\title{
Desmoplakin mutation in neonatal erythroderma
}

Katariina Hannula-Jouppi11, 2, 3, Svetlana Vakkilainen ${ }^{4,2}$, Laura Puhakka ${ }^{4,2}$, Harri Saxen ${ }^{4,2}$, Annamari Ranki ${ }^{1,2}$

1. Department of Dermatology, Helsinki University Hospital, Finland. 2. University of Helsinki, Finland. 3. Folkhälsan Institute of Genetics, Helsinki, Finland. 4. Children's hospital, Helsinki University Hospital, Finland.

katarina.hannula-jouppi@hus.fi

\section{INTRODUCTION}

A novel Desmoplakin (DSP) heterozygous mutation, c. $1756 \mathrm{C}>\mathrm{T}$, p.(His586Tyr), was found in an infant with ichthyosiform erythroderma, recurrent sepsis and skin infections, failure to thrive, constant vomiting and brain abnormalities. A similar phenotype named SAMsyndrome (Severe dermatitis, multiple Allergies and Metabolic wasting) was has been reported in five cases with DSG1 mutations and one case with a neighboring $D S P$ mutation c.1757C >T, p.(His586Pro)1,2,3. Our patient presented with some novel features e.g. brain abnormalities and recurrent severe HSV stomatitis

\section{METHODS}

Immunohistochemistry on skin biopsies with LEKTI, Filaggrin, DSP and electron microscopy (EM) were done to study epidermal function and structure.

Detailed phenotypic, vast immunological and metabolic analyses and radiologic imaging were conducted.

Icthyosis and immunodeficiency gene panels and whole exome sequencing (WES) were performed to detect the genetic cause.

A literature review of previously reported $D S P$ and $D S G 1$ patients with a similar phenotype with congenital erythroderma was done.

\begin{tabular}{|c|c|c|c|c|c|c|c|c|}
\hline Reference & Here & 1 & 2 & 2 & 2 & 3 & 3 & \\
\hline Gene & \multicolumn{2}{|c|}{$D S P$} & \multicolumn{5}{|c|}{$\overline{\mathrm{DSG} 1}$} & \\
\hline Mutation & c. $1756 \mathrm{C}>\mathrm{T}$ & $\begin{array}{c}\text { c. } 1757 \mathrm{~A}> \\
\text { C }\end{array}$ & $\begin{array}{l}\text { C. } 49- \\
1 G>A\end{array}$ & $\begin{array}{l}\text { C. } 49- \\
1 G>A\end{array}$ & c. $1861 \mathrm{delG}$ & $\begin{array}{l}\text { c.1892- } \\
1 \text { delG }\end{array}$ & $\begin{array}{l}\text { c.1892- } \\
1 \text { delG }\end{array}$ & \\
\hline Age & 13 months & 6 years & 7 years & 3 years & 9 months & 7 years & 13 years & \\
\hline Congenital ichtyosis & + & + & + & + & + & + & + & $7 / 7$ \\
\hline PPK & - & + & + & + & - & + & + & $5 / 7$ \\
\hline Hypotrichosis & + & + & + & + & + & - & - & $5 / 7$ \\
\hline Curly hair & - & - & - & - & - & + & + & $2 / 7$ \\
\hline Multiple allergies & - & + & + & + & + & + & $\mathrm{N} / \mathrm{A}$ & $5 / 7$ \\
\hline Recurrent infections & + & + & + & + & + & + & - & $6 / 7$ \\
\hline Recurrent sepsis & + & + & - & - & - & - & - & $2 / 7$ \\
\hline Failure to thrive & + & + & + & + & + & + & - & $6 / 7$ \\
\hline $\begin{array}{l}\text { Gastrointestinal } \\
\text { problems }\end{array}$ & + & + & + & + & - & - & $\mathrm{N} / \mathrm{A}$ & $4 / 7$ \\
\hline Developmental delay & + & + & + & + & + & $\mathrm{N} / \mathrm{A}$ & - & $5 / 7$ \\
\hline IgE IU/ml elevated & 985 & 611 & 10,900 & 266 & 1,438 & $>25,000$ & $\mathrm{~N} / \mathrm{A}$ & $6 / 7$ \\
\hline Hypoalbunemia & + & $\mathrm{N} / \mathrm{A}$ & + & + & + & $\mathrm{N} / \mathrm{A}$ & $\mathrm{N} / \mathrm{A}$ & $4 / 7$ \\
\hline Hypernatremia & + & $\mathrm{N} / \mathrm{A}$ & + & + & $\mathrm{N} / \mathrm{A}$ & $\mathrm{N} / \mathrm{A}$ & $\mathrm{N} / \mathrm{A}$ & $3 / 7$ \\
\hline
\end{tabular}

\section{CONCLUSIONS}

$D S P$ mutations should be considered in erythrodermic infants, with recurrent sepsis and infections, developmental delay and failure to thrive. DSP and DSG1 mutations can cause a similar phenotype and lead to severe skin barrier defects resulting in a Netherton syndrome like phenotype.

\section{OBJECTIVES}

1. Evaluate the phenotypic effect of a novel $D S P$ mutation.

2. Compare phenotypes with the one other $D S P$ heterozygous mutation patient and five patients with homozygous DSG1 mutations with similar phenotypes.

\section{RESULTS}

An otherwise healthy boy presented at 2 weeks age with a seborrheic like eczema, which progressed into an ichtyosiform eryhtroderma with recurrent enterococcal and staphylococcal sepsis, skin infections, severe failure to thrive, constant vomiting and developmental delay.

MRI showed brain abnormalities and he suffered from recurrent HSV stomatitis. He had persistent thrombocytosis, hypoalbunemia, hypernatremia and elevated $\lg E$ levels without eosinophilia. A non-lgE mediated cow's milk allergy was diagnosed. Vast immunologic and metabolic analyses were normal.

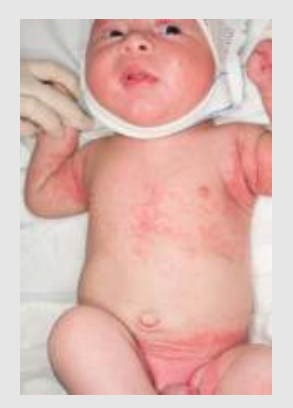

3 weeks

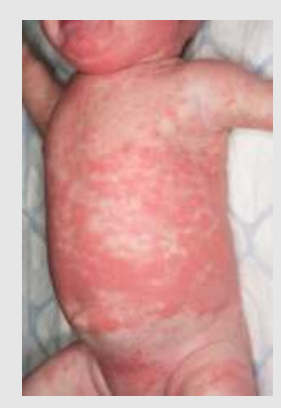

2 months

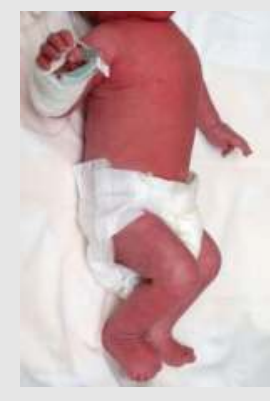

4 months

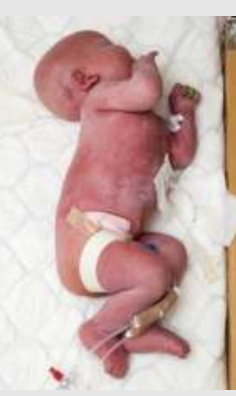

6 months 11 months

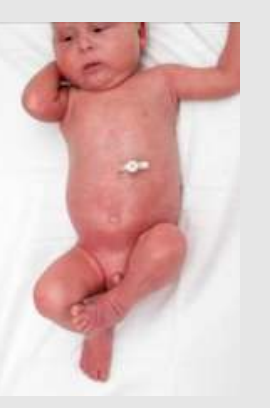

control

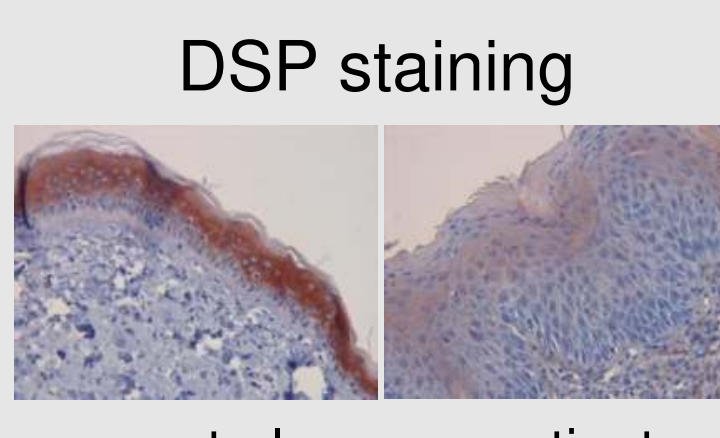

patient
Skin biopsy showed a psoriformic reaction with absent filaggrin but nomal LEKTI staining. WES revealed a de novo heterozygous $D S P$ missense variant $c .1756 \mathrm{C}>\mathrm{T}$, p.(His586Tyr) predicted deleterious by in silico tools SIFT and MutationTaster. Loss of DSP was seen in skin samples and electron microscopy revealed significant loss of intercellular cohesiveness and decreased numbers of desmosomes and absent tonofilaments perinuclearly. IVIG and acitretin treatment and optimizing enteral nutrition alleviated scaling, erythema and itch and induced hair regrowth.

$D S P$ mutation patients suffered from recurrent sepsis. Otherwise clinical features were similar with DSP and DSG1 mutations.

\section{REFERENCES}

1. McAleer MA et al. J Allergy Clin Immunol 2015,136:1268-76.

2. Samuelov L, et al. Nat Genet 2013,10:1244-8.

3. Cheng R, et al. J Dermatol. 2016,43:1201-4. 